\title{
Postoperative Outcomes in Exploratory Laparotomy and Intestinal Resection in Children: A Secondary Descriptive Observational Analysis
}

\author{
Claudine Kumba \\ Department of Pediatric Anesthesia and Critical Care, Necker Enfants Malades University Hospital, Assistance Publique \\ Hôpitaux de Paris, APHP, University of Paris, Paris, France \\ Email: claudine.kumba@gmail.com, claudine.kumba@aphp.fr
}

How to cite this paper: Kumba, C. (2021) Postoperative Outcomes in Exploratory Laparotomy and Intestinal Resection in Children: A Secondary Descriptive Observational Analysis. Open Journal of Pediatrics, 11, 618-626.

https://doi.org/10.4236/ojped.2021.114057

Received: October 9, 2021

Accepted: November 14, 2021

Published: November 17, 2021

Copyright $\odot 2021$ by author(s) and Scientific Research Publishing Inc. This work is licensed under the Creative Commons Attribution International License (CC BY 4.0).

http://creativecommons.org/licenses/by/4.0/

(c) (i) Open Access

\begin{abstract}
Background: We previously reported independent predictors of intraoperative and postoperative morbidity. These were age, American Society of Anesthesiologists Score (ASA), emergency situations, surgery and transfusion. ASA was the independent predictor of mortality. We conducted a secondary analysis of this previous retrospective study in patients who underwent exploratory laparotomy and intestinal resection. Objectives: The objective was to describe intraoperative and postoperative outcomes in patients who underwent exploratory laparotomy and intestinal resection in the initial study and to present a research protocol for intraoperative and postoperative optimization. Methods: Secondary analysis of the initial study was used. The Ethics Committee approved the study. Results: There were 54 patients with a median age of 15.5 [0 - 172] months. Thirty-seven (68.5\%) patients underwent intestinal resection, nine (16.7\%) underwent exploratory laparotomy, and eight $(16.8 \%)$ underwent laparotomy for volvulus. Fourteen $(25.9 \%)$ patients had intraoperative and/or postoperative complications. Two (3.7\%) patients had an intraoperative hemorrhagic shock. Two (3.7\%) patients had a postoperative cardio-circulatory failure. Three $(5.6 \%)$ had postoperative respiratory failure. One $(1.8 \%)$ patient had postoperative multiple organ failure and neurologic failure. Three (5.6\%) patients had postoperative abdominal sepsis. One $(1.8 \%)$ patient had postoperative multiple organ sepsis and neuromeningeal sepsis. Four (7.4\%) patients had postoperative pulmonary sepsis. Two (3.7\%) had postoperative septicemia. Six (11.1\%) patients had reoperations. Seventeen $(31.5 \%)$ patients had an intraoperative transfusion. The in-hospital mortality rate was $3.7 \%$ in two patients. Conclusion: The number
\end{abstract}


of patients with postoperative complications in this cohort was not negligible. We, therefore, elaborated a research protocol where intraoperative patient management will be guided with transthoracic echocardiography for fluid and hemodynamic therapy optimization. The objective of this study protocol is to clarify the impact of intraoperative goal-directed fluid and hemodynamic therapy with transthoracic echocardiography on postoperative outcomes in terms of complications in pediatric surgical patients.

\section{Keywords}

Exploratory Laparotomy, Intestinal Resection, Children, Intraoperative and Postoperative Outcomes, Intraoperative Goal-Directed Therapies

\section{Introduction}

Intraoperative and postoperative outcomes in children have been reported to be multifactorial [1] [2] [3] [4]. Reported independent predictors of intraoperative and postoperative complications were age, American Society of Anesthesiologists Score (ASA), emergency situations, surgery and transfusion [1] [2] [3] [4]. ASA was the independent predictor of mortality [1].

Intraoperative and postoperative complications in pediatric surgical settings with regard to age have been described previously [5]-[10]. When considering the entire initial cohort of 594 patients, the overall rate of patients with intraoperative and/or postoperative complications was $23.9 \%$ [1]. The most-reported intraoperative complication was hemorrhagic shock, with an overall rate of $3.9 \%$ [1]. The most commonly reported postoperative organ failure was neurologic, followed by respiratory, cardio-circulatory and multiple organ failure, with overall rates of $4.2 \%, 3.5 \%, 3 \%$ and $1.5 \%$, respectively [1]. The most commonly reported postoperative infection was septicemia, followed by pulmonary sepsis, abdominal sepsis, surgical wound sepsis and urinary sepsis, with overall rates of $3.7 \%, 2.9 \%, 2.7 \%, 2 \%$ and $1.3 \%$, respectively [1]. Overall transfusion rate was $49.2 \%$. Overall rate of reoperation was $7.2 \%$. The rate of emergency interventions was $22.9 \%$. Overall in-hospital mortality rate was $1.9 \%$.

We conducted a secondary analysis of this initial cohort with the primary objective of describing intraoperative and postoperative outcomes in patients who underwent exploratory laparotomy and intestinal resection. The secondary objective was to propose and implement intraoperative optimization management research protocols for postoperative outcome improvement in these pediatric surgical settings.

\section{Methods and Materials}

A secondary analysis of patients who underwent exploratory laparotomy and intestinal resection was included in the initial study [1]. In the initial study, we emphasized on determining predictors of intraoperative and postoperative out- 
comes. In this secondary analysis we aimed to emphasize on the specific intraoperative and postoperative outcomes in a specific surgery, namely laparotomy.

Outcomes were defined in terms of organ dysfunction, infection or sepsis, length of stay in the intensive care unit, length of hospital stay, length of total hospital stay (length of intensive care unit stay and length of stay in the standard hospitalization ward), duration of mechanical ventilation and transfusion.

Organ dysfunction and sepsis were defined per system with clinical, laboratory and imaging findings as a state of organ alteration not present in the preoperative period or present preoperatively with postoperative majoration or increase. Multiple organ dysfunction or multiple organ sepsis was defined as a state of more than one organ alteration with clinical, laboratory, and/or imaging findings.

The study was declared to the National Commission for Computer Science and Liberties (CNIL) under registration number 2028257 v0 on 21 February 2017 and approved by the Ethics Committee of Necker under registration number 2017-CK-5-R1 on 21 March 2017.

The inclusion criteria were patients who underwent exploratory laparotomy or intestinal resection and those aged less than 18 years old included in the initial study.

The exclusion criteria were patients who did not undergo exploratory laparotomy or intestinal resection and were aged more than 18 years old.

Patients were included retrospectively from 1 January 2014 to 17 May 2017.

Statistics were analyzed with XLSTAT 2020.4.1. software. Continuous variables were expressed as medians with ranges or means with standard deviations. Category variables were described in proportions.

\section{Results}

Table 1 illustrates general characteristics.

There were 54 patients with a median age of 15.5 [0 - 172] months and a median weight of 6.4 [1.4 - 42] kilograms. There were four, twenty-four, twenty-two and four American Society of Anesthesiologists grade 1, 2, 3 and 4 patients, respectively. Thirty-two patients had an emergent intervention. Fourteen patients had intraoperative and/or postoperative complications. Two patients had intraoperative hemorrhagic shock. Two patients had a postoperative cardio-circulatory failure. Three had postoperative respiratory failure. One patient had postoperative multiple organ failure and neurologic failure. Three patients had postoperative abdominal sepsis. One patient had postoperative multiple organ sepsis and neuromeningeal sepsis. Four patients had postoperative pulmonary sepsis. Two had postoperative septicemia. Six patients had reoperations. Seventeen patients had intraoperative transfusion. There were two in-hospital deaths. All patients with fatal outcomes had comorbidity, namely, congenital heart disease and all were managed on an emergency basis.

Table 2 illustrates the characteristics of patients with fatal outcomes.

Table 3 illustrates surgery. 
Table 1. General characteristics.

\begin{tabular}{|c|c|}
\hline Characteristic & $\mathrm{N}=54$ \\
\hline Median age [range] in months & $15.5[0-172]$ \\
\hline Median weight [range] in kilograms & $6.4[1.4-42]$ \\
\hline ASA I n (\%) & $4(7.4)$ \\
\hline ASA II n (\%) & $24(44.4)$ \\
\hline ASA III n (\%) & $22(40.7)$ \\
\hline ASA IV n (\%) & $4(7.4)$ \\
\hline Emergency surgery n (\%) & $32(59.3)$ \\
\hline Elective surgery n (\%) & $22(40.7)$ \\
\hline Re-operation n (\%) & $6(11.1)$ \\
\hline $\begin{array}{l}\text { Patients with intra-operative and or postoperative } \\
\text { complications (organ failure or sepsis) } \mathrm{n}(\%)\end{array}$ & $14(25.9)$ \\
\hline Intraoperative hemorrhagic shock n (\%) & $2(3.7)$ \\
\hline Postoperative cardio-circulatory failure n (\%) & $2(3.7)$ \\
\hline Postoperative respiratory failure $\mathrm{n}(\%)$ & $3(5.6)$ \\
\hline Postoperative multiple organ failure $\mathrm{n}(\%)$ & $1(1.8)$ \\
\hline Postoperative neurologic failure $\mathrm{n}(\%)$ & $1(1.8)$ \\
\hline Postoperative abdominal sepsis n (\%) & $3(5.6)$ \\
\hline Postoperative multiple organ sepsis n (\%) & $1(1.8)$ \\
\hline Postoperative neuro-meningeal sepsis n (\%) & $1(1.8)$ \\
\hline Postoperative pulmonary sepsis n (\%) & $4(7.4)$ \\
\hline Postoperative septicemia n (\%) & $2(3.7)$ \\
\hline In-hospital mortality n (\%) & $2(3.7)$ \\
\hline Transfusion $\mathrm{n}(\%)$ & $17(31.5)$ \\
\hline Median packed red blood cells volume in $\mathrm{ml}$ [range] & $0[0-5]$ \\
\hline Median fresh frozen plasma volume in $\mathrm{ml}$ [range] & $0[0-2]$ \\
\hline Median concentrated platelet units [range] & $0[0-2]$ \\
\hline Mean preoperative hemoglobin levels \pm standard deviation in $\mathrm{g} / \mathrm{dl}$ & $12.6 \pm 3.3$ \\
\hline Mean postoperative hemoglobin levels \pm standard deviation in $\mathrm{g} / \mathrm{dl}$ & $11.3 \pm 1.9$ \\
\hline Median crystalloid volume in $\mathrm{ml}$ [range] & $1287.5[60-3100]$ \\
\hline Median colloid volume in $\mathrm{ml}$ [range] & $0[0-1000]$ \\
\hline Median length of intensive care unit stay in days [range] & $5[0-77]$ \\
\hline Median length of hospital stay in days [range] & $7[0-101]$ \\
\hline Median total length of hospital stay in days [range] & $15[4-178]$ \\
\hline Median total length of mechanical ventilation in days [range] & $0[0-28]$ \\
\hline
\end{tabular}


Table 2. Patients with fatal outcome.

\begin{tabular}{cccccccc}
\hline Surgery & $\begin{array}{c}\text { Age in } \\
\text { days }\end{array}$ & $\begin{array}{c}\text { ASA } \\
\text { score }\end{array}$ & Co-morbidities $\begin{array}{c}\text { Intra-operative } \\
\text { complications }\end{array}$ & $\begin{array}{c}\text { Postoperative } \\
\text { outcome }\end{array}$ & $\begin{array}{c}\text { Delay of } \\
\text { in-hospital } \\
\text { mortality } \\
\text { in days }\end{array}$ \\
\hline $\begin{array}{l}\text { Laparotomy } \\
\text { for volvulus }\end{array}$ & 30 & 4 & $\begin{array}{c}\text { Congenital } \\
\text { Heart Disease }\end{array}$ & 0 & Multiple Organ Sepsis & 75 & 1 \\
$\begin{array}{l}\text { Intestinal } \\
\text { Resection }\end{array}$ & $<28$ & 3 & $\begin{array}{c}\text { Congenital } \\
\text { Heart Disease }\end{array}$ & 0 & $\begin{array}{c}\text { Multiple Organ Failure } \\
\text { and Neuro-meningeal } \\
\text { sepsis }\end{array}$ & 63 & 1 \\
\hline
\end{tabular}

Table 3. Surgery.

\begin{tabular}{cc}
\hline Surgery & Number of (\%) \\
\hline Intestinal resection & $37(68.5)$ \\
Exploratory laparotomy & $9(16.7)$ \\
Laparotomy for Volvulus & $8(14.8)$ \\
\hline
\end{tabular}

Thirty-seven patients underwent intestinal resection, nine underwent exploratory laparotomy, and eight underwent laparotomy for volvulus.

Table 4 illustrates the co-morbidities.

The most common comorbidities were congenital coagulation disorders, congenital heart disease, necrotizing enterocolitis, cancer, Hirschsprung's disease, hepatic failure, intestinal pseudoocclusion, neurofibromatosis, and preterm births.

\section{Discussion}

The rate of patients with intraoperative and/or postoperative complications in this secondary analysis was comparable to that of the initial cohort [1] [2] [3] [4]. This was not surprising since this cohort was a sub-cohort of the initial study. The results of this secondary analysis highlight that morbidity in terms of postoperative organ dysfunction and infection in patients who underwent exploratory laparotomy and intestinal resection was high. Similar analyses have been conducted in other surgical pediatric settings [5]-[15]. How could these outcomes be improved? We conceptualized a research protocol where intra-operative fluid and hemodynamic therapy will be guided with transthoracic echocardiography to optimize intraoperative patient status [16] [17]. Echocardiography has been validated in several pediatric studies to guide fluid therapy in children [18] [19] [20] [21] [22]. The objectives of intraoperative goal-directed fluid and hemodynamic therapy are to optimize patient status to improve postoperative outcome [23] [24]. In adults, goal-directed fluid and hemodynamic therapy has been shown to reduce postoperative morbidity, mortality and length of hospital stay in surgical patients [25]. In children, the impact of goal directed-fluid and hemodynamic therapy has not yet been demonstrated. Our hypothesis is that goal 
Table 4. Co-morbidities.

\begin{tabular}{|c|c|}
\hline Co-morbidity & Number of patients (\%) \\
\hline Bronchodysplasia & $1(1.8)$ \\
\hline Cancer & $3(5.6)$ \\
\hline Congenital coagulation disorder & $10(18.5)$ \\
\hline Congenital heart disease & $4(7.4)$ \\
\hline Crohn's disease & $1(1.8)$ \\
\hline Duodenal atresia & $1(1.8)$ \\
\hline Former preterm & $1(1.8)$ \\
\hline Hepatic failure & $2(3.7)$ \\
\hline Hepatoblastoma & $1(1.8)$ \\
\hline Hirschprung & $3(5.6)$ \\
\hline Intestinal pseudo-occlusion & $2(3.7)$ \\
\hline Necrotizing enterocolitis & $4(7.4)$ \\
\hline Neurofibromatosis & $2(3.7)$ \\
\hline Polymalformation syndrome & $1(1.8)$ \\
\hline Polytrauma & $1(1.8)$ \\
\hline Pre-term & $2(3.7)$ \\
\hline Pre-term + Necrotizing enterocolitis & $2(3.7)$ \\
\hline Transplantation & $1(1.8)$ \\
\hline
\end{tabular}

directed therapy with validated tools in children like echocardiography could improve postoperative outcome in pediatric surgical patients, too [16] [17]. In our hospital goal-directed fluid and hemodynamic therapy with transthoracic echocardiography is not yet a routine practice in major surgery. This research protocol will clarify the impact of intraoperative echocardiography for fluid and hemodynamic therapy on postoperative outcome in surgical pediatric patients.

One-third of the patients in this subcohort were transfused. Transfusion is also an important aspect of intraoperative management. Transfusion has been shown to be an independent predictor of postoperative morbidity [1] [2] [3] [4]. Optimizing intraoperative transfusion with point-of-care viscoelastic methods has been shown to reduce transfusion requirements and decrease the length of hospital stay in pediatric hemorrhagic surgical settings [26]. Goal-directed therapies include fluid and hemodynamic therapy with validated tools in children, transfusion goal-directed protocols guided with point-of-care tests. These goal-directed therapies have in common the aim of optimizing the relationship between oxygen consumption and oxygen delivery, to avoid situations where oxygen consumption becomes dependent on oxygen delivery [23] [24]. Situations, where oxygen consumption becomes oxygen delivery dependent, predispose patients to 
organ dysfunction [23] [24]. Oxygen delivery is determined among other factors, by cardiac output and hemoglobin levels. Goal directed fluid and hemodynamic therapy with echocardiography has the objective of optimizing cardiac output which is a determinant of oxygen delivery [23] [24]. Transfusion protocols guided with point-of-care tests have the objective of optimizing blood product administration in order to transfuse the right product at the right time. Optimal hemoglobin levels are necessary for an optimal oxygen consumption-oxygen delivery relation [23] [24]. Our hypothesis is that intraoperative optimization with goal-directed therapies could contribute to upgrading postoperative outcomes in these major surgical settings. To date, goal-directed therapies are not well developed in children. A systematic review and meta-analysis in children revealed that indirect non-optimal biomarkers of oxygen consumption-oxygen delivery relation namely lactate levels, regional oxygen saturation, mixed venous oxygen saturation were predictors of adverse postoperative outcomes in major pediatric surgical patients in terms of morbidity; mortality and length of hospital stay [27]. We have elaborated a study protocol with transthoracic echocardiography for intraoperative fluid and hemodynamic therapy optimization. This protocol will clarify the impact of this validated tool on postoperative outcome in pediatric surgical patients [16] [17]. Similar conclusions have been drawn in other major pediatric surgical settings where goal-directed therapies need to be developed in research protocols to determine their impact on postoperative outcomes [11] [12] [13] [14] [15].

\section{Conclusion}

The number of patients with postoperative complications in this cohort was $25.9 \%$. To improve these outcomes, we have elaborated a research protocol with transthoracic echocardiography. Intraoperative goal-directed therapies need to be developed in research protocols in these major pediatric surgical settings to determine their impact on intraoperative and postoperative outcomes.

\section{Author' Contributions}

Claudine Kumba conceptualized and designed the study and drafted the initial manuscript. She designed the data collection instruments, collected data, carried out initial and final analyses.

\section{Presentation of Preliminary Results}

This manuscript was registered as a preprint under the DOI number https://doi.org/10.21203/rs.3.rs-785514/v3 on Research Square, a preprint platform.

\section{Ethics Approval}

This study received approval from the Ethics Committee of Necker on 21 March 2017 under registration number 2017-CK-5-R1 and waived patient consent. 


\section{Conflicts of Interest}

The author declared no conflicts of interest regarding the publication of this paper.

\section{References}

[1] Kumba, C., Cresci, F., Picard, C., et al. (2017) Transfusion and Morbi-Mortality Factors: An Observational Descriptive Retrospective Pediatric Cohort Study. Journal of Anesthesia \& Critical Care: Open Access, 8, Article No. 00315. https://doi.org/10.15406/jaccoa.2017.08.00315

[2] Kumba, C., Lenoire, A., Cairet, P., Dogaru-Dedieu, E., Belloni, I. and Orliaguet, G. (2018) Is Transfusion an Independent Risk Factor of Postoperative Outcome in Pediatric Orthopedic Surgical Patients? A Retrospective Study. Journal of Emergency Medicine \& Critical Care, 4, Article No. 7. https://doi.org/10.13188/2469-4045.1000018

[3] Kumba, C., Querciagrossa, S., Thomas, B. and Treluyer, J.M. (2018) Transfusion and Postoperative Outcome in Pediatric Abdominal Surgery. Journal of Clinical Research in Anesthesiology, 1, 1-8. https://doi.org/10.33309/2639-8915.010103

[4] Kumba, C., Taright, H., Terzi, E., Telion, C., Beccaria, K., Paternoster, G., Zerah, M., Bustarret, O., Jugie, M., Rubinsztajn, R. and Treluyer, J.M. (2018) Blood Product Transfusion and Postoperative Outcome in Pediatric Neurosurgical Patients. EC Anaesthesia, 4, 288-298.

[5] Kumba, C. (2021) Preterm Infants in Major Abdominal Surgery and Postoperative Outcome. Research Square. https://doi.org/10.21203/rs.3.rs-669064/v1

[6] Kumba, C. (2021) Postoperative Outcome in Non-Preterm Infants under One Year Old in Non-Cardiac Surgery. Research Square. https://doi.org/10.53902/SOJPCN.2021.01.000509

[7] Kumba, C. (2021) Children Aged between 1 and 3 Years in Noncardiac Surgery and Postoperative Outcome. EC Paediatrics, 10, 67-74.

[8] Kumba, C. (2021) Postoperative Outcome in Children Aged between 3 and 6 Years in Abdominal Surgery, Neurosurgery and Orthopedics. Pediatric Anesthesia and Critical Care Journal, 9, 43-47.

[9] Kumba, C. (2021) Postoperative Outcome in Children Aged between 6 and 10 Years in Major Abdominal Surgery, Neurosurgery and Orthopedic Surgery. Research Square. https://doi.org/10.21203/rs.3.rs-669076/v1

[10] Kumba, C. (2021) Major Abdominal Surgery, Neurosurgery, Orthopedic Surgery in Children Aged between 10 and 18 Years and Postoperative Outcome. SOJ Pediatrics and Clinical Neonatology, 1, 1-7. https://doi.org/10.53902/SOJPCN.2021.01.000509

[11] Kumba, C. (2021) Liver Transplantation in Children and Impact of Intraoperative Goal-Directed Therapies on Postoperative Outcome. Research Square. https://doi.org/10.21203/rs.3.rs-744584/v1

[12] Kumba, C. (2021) Scoliosis in Children: Impact of Goal Directed Therapies on Intraoperative and Postoperative Outcomes. Research Square. https://doi.org/10.21203/rs.3.rs-765785/v1

[13] Kumba, C. (2021) Patient Blood Management in Craniosynostosis Surgery. Research Square. https://doi.org/10.21203/rs.3.rs-774234/v1

[14] Kumba, C. (2021) Intraoperative Goal-Directed Therapies in Femoral and Pelvic Osteotomies in Children and In-Hospital Postoperative Outcomes. Research Square. 
https://doi.org/10.21203/rs.3.rs-777279/v1

[15] Kumba, C. (2021) Neuroblastoma in Children: Intraoperative Goal Directed Therapy, Intraoperative and Postoperative Outcomes. Research Square. https://doi.org/10.21203/rs.3.rs-785499/v1

[16] Kumba, C. (2020) Goal Directed Fluid and Hemodynamic Therapy and Postoperative Outcomes in Children: Value of Transthoracic Echocardiographic Aortic Blood Flow Peak Velocity Variation: A Multi-Centre Randomized Controlled Trial Protocol. Advances in Pediatric Research, 7, Article No. 35.

https://doi.org/10.35248/2385-4529.20.7.35

[17] Kumba, C. (2019) Do Goal Directed Therapies Improve Postoperative Outcome in Children? (Perioperative Goal Directed Fluid and Hemodynamic Therapy; Transfusion Goal Directed Therapy Using Viscoelastic Methods and Enhanced Recovery after Surgery and Postoperative Outcome): A Study Research Protocol. Acta Scientific Paediatrics, 2, 17-19. https://doi.org/10.31080/ASPE.2019.02.0094

[18] Tibby, S.M., Durward, A. and Murdoch, I.A. (2001) Are Transoesophageal Doppler Parameters a Reliable Guide to Paediatric Haemodynamic Status and Fluid Management? Intensive Care Medicine, 27, 201-205. https://doi.org/10.1007/s001340000795

[19] Murdocc, I.A., Marsh, M.K., Tibby, S.M. and McLuckie, A. (1995) Continuous Haemodynamic Monitoring in Children: Use of Transoesophageal Doppler. Acta Paediatrica, 84, 761-764. https://doi.org/10.1111/j.1651-2227.1995.tb13751.x

[20] Weber, T., Wagner, T., Neumann, K. and Deusch, E. (2015) Low Predictability of Three Different Noninvasive Methods to Determine Fluid Responsiveness in Critically Ill Children. Pediatric Critical Care Medicine, 16, e89-e94. https://doi.org/10.1097/PCC.0000000000000364

[21] Gan, H., Cannesson, M., Chandler, J.R. and Ansermino, J.M. (2013) Predicting Fluid Responsiveness in Children: A Systematic Review. Anesthesia \& Analgesia, 117, 1380-1392. https://doi.org/10.1213/ANE.0b013e3182a9557e

[22] Pereira de Souza Neto, E., Grousson, S., Duflo, F., et al. (2011) Predicting Fluid Responsiveness in Mechanically Ventilated Children under General Anaesthesia Using Dynamic Parameters and Transthoracic Echocardiography. British Journal of Anaesthesia, 106, 856-864. https://doi.org/10.1093/bja/aer090

[23] Kumba, C. (2020) Physiology Principles Underlying Goal Directed Therapies in Children. Research in Pediatrics \& Neonatology, 4, RPN.000591.2020. https://doi.org/10.31031/RPN.2020.04.000591

[24] Kumba, C. (2020) Rationale of Goal Directed Therapies in Children. Advances in Pediatric Research, 7, 42. https://doi.org/10.35248/2385-4529.20.7.35

[25] Chong, M., Wang, Y., Berbenetz, N., et al. (2018) Does Doal-Directed Haemodynamic and Fluid Therapy Improve Peri-Operative Outcomes? A Systematic Review and Meta-Analysis. European Journal of Anaesthesiology, 35, 469-483. https://doi.org/10.1097/EJA.0000000000000778

[26] Kumba, C., Querciagrossa, S., Harte, C., Willems, A., et al. (2019) A Systematic Review and Meta-Analysis of Goal Directed Intra-Operative Transfusion Protocols Guided by Viscoelastic Methods and Perioperative Outcomes in Children. International Journal of Recent Scientific Research, 10, 31466-31471.

[27] Kumba, C., Willems, A., Querciagrossa, S., et al. (2019) A Systematic Review and Meta-Analysis of Intraoperative Goal Directed Fluid and Haemodynamic Therapy in Children and Postoperative Outcome. Journal of Emergency and Critical Care Medicine, 5, Article No. 9. https://doi.org/10.13188/2469-4045.1000020 